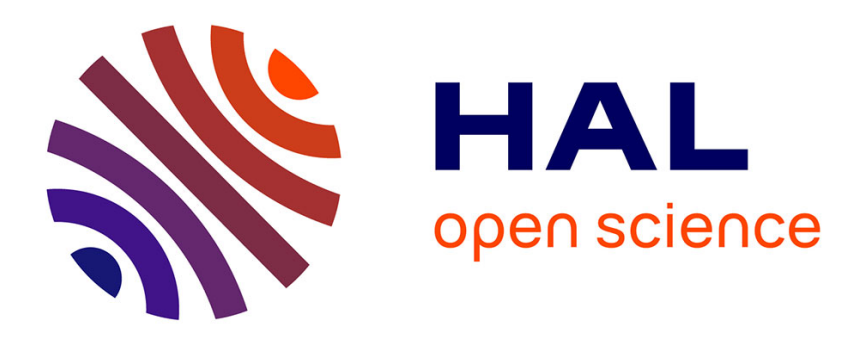

\title{
Spectres d'absorption du nitrate de potassium dans l'infrarouge lointain
}

\author{
F. Brehat, A. Hadni
}

\section{To cite this version:}

F. Brehat, A. Hadni. Spectres d'absorption du nitrate de potassium dans l'infrarouge lointain. Revue de Physique Appliquée, 1975, 10 (2), pp.47-50. 10.1051/rphysap:0197500100204700 . jpa-00243879

\section{HAL Id: jpa-00243879 https://hal.science/jpa-00243879}

Submitted on 1 Jan 1975

HAL is a multi-disciplinary open access archive for the deposit and dissemination of scientific research documents, whether they are published or not. The documents may come from teaching and research institutions in France or abroad, or from public or private research centers.
L'archive ouverte pluridisciplinaire HAL, est destinée au dépôt et à la diffusion de documents scientifiques de niveau recherche, publiés ou non, émanant des établissements d'enseignement et de recherche français ou étrangers, des laboratoires publics ou privés. 


\title{
REVUE DE PHYSIQUE APPLIQUÉE
}

Supplément au « Journal de Physique *

\author{
Classification \\ Physics Abstracts \\ 8.822

\section{SPECTRES D'ABSORPTION DU NITRATE DE POTASSIUM DANS L'INFRAROUGE LOINTAIN}

\author{
F. BREHAT et A. HADNI \\ Institut de Physique (*), Université de Nancy I, 54000 Nancy, France
}

(Reçu le 2 août 1974)

\begin{abstract}
Résumé. - Le spectre d'absorption dans l'infrarouge lointain d'un monocristal de nitrate de potassium en lumière polarisée ne montre aucune différence entre les 2 phases rhomboédriques l'une paraélectrique, l'autre ferroélectrique qui correspondent toutes deux à une unité formulaire dans la maille. La vibration de translation des ions $\mathrm{NO}_{3}^{-}$et $\mathrm{K}^{+}$perpendiculaire à l'axe $c$ donne une large bande d'absorption pour $E / / c$ qui ne se déplace pas avec la température, et n'apporte donc aucun argument en faveur d'une transition displacive. Aucune absorption supplémentaire n'apparaît dans la phase paraélectrique où elle aurait indiqué l'apparition d'un certain désordre. Par contre conformément à la théorie des groupes, des raies nouvelles apparaissent dans la phase orthorhombique où la maille contient 4 unités formulaires, mais on observe moins de raies qu'on n'en attend.
\end{abstract}

\begin{abstract}
The far infra-red absorption spectra of a potassium nitrate single crystal, using polarized light, show no difference between the paraelectric and ferroelectric phases, both of which are rhombohedral with one formula unit per unit cell. The translational vibration of the $\mathrm{NO}_{3}^{-}$and $\mathrm{K}^{+}$ions perpendicular to the $c$-axis yields a wide absorption band parallel to $c$ which does not shift with temperature and thus does not show evidence of a displacive transition. No additional absorption (which would have shown the onset of some disorder) appears in the paraelectric phase. Nevertheless, in agreement with group theory, new lines appear in the low temperature orthorhombic phase having 4 formula units per unit cell, although several expected lines are not seen.
\end{abstract}

1. Introduction. - On sait depuis longtemps [1] faire pousser des cristaux orthorhombiques de nitrate de potassium par évaporation lente d'une solution saturée. Il s'agit de la phase II paraélectrique. Lorsqu'on chauffe le cristal on constate à $399 \mathrm{~K}$ la transformation en phase I rhomboédrique. Sawada et ses coll. ont montré en 1958 [2] que si l'on refroidit lentement le cristal on ne retrouve pas la phase orthorhombique à $399 \mathrm{~K}$. On observe toutefois à $398 \mathrm{~K}$ une transformation mineure qui conserve la structure rhomboédrique mais fait apparaître des propriétés ferroélectriques (phase III). Cette phase est stable dans un étroit domaine de température $(10 \mathrm{~K})$ limité à $388 \mathrm{~K}$ par un point de transformation où l'on retrouve la phase II orthorhombique qui reste stable jusqu'aux plus basses températures. Le tableau I résume les données cristallographiques sur les 3 phases. Pour Tahvonen [3] la

(*) Equipe de recherche associée au C. N. R.S. n 14. transition I $\rightarrow$ III à $398 \mathrm{~K}$ serait du type déplacement et concernerait l'ion $\mathrm{NO}_{3}^{-}$. Pour Shinnaka [4] la transition serait du type ordre-désordre. La spectroscopie a déjà apporté des renseignements intéressants. La diffusion Raman d'un monocristal étudiée par Teng [5, 6] a montré que les phases I et III présentaient des spectres identiques correspondant au même groupe d'espace $C_{3 v}^{5}$. Les spectres infrarouges n'ont été étudiés qu'avec des échantillons polycristallins, par absorption pour les hautes fréquences $[7,8,9]$ et par réflexion pour les basses fréquences [10]. Dans l'infrarouge lointain, en lumière polarisée, nous connaissons toutefois le spectre de réflexion infrarouge dans la phase $I$ de 50 à $250 \mathrm{~cm}^{-1}$ réalisé par Grynwald et cité par Teng [5].

2. Expérimentation. - Le cristal obtenu par évaporation lente d'une solution saturée se présente dans la phase II orthorhombique et il est facile de repérer l'axe $c$, et de tailler des lames d'orientation convenable dont l'épaisseur est de l'ordre de $30 \mu \mathrm{m}$. L'étude du 
TABLEAU I

Structures des trois phases du nitrate de potassium

Phase
II

3. Résultats et discussion. - Les figures 1 et 2 et le tableau II résument notre étude.

3.1 DÉNOMBREMENT DES VIBRATIONS ACTIVES EN INFRAROUGE. - Il semble bien établi que les structures rhomboédriques du nitrate de potassium dans les phases I et III sont bien déç̣ites par le même groupe de

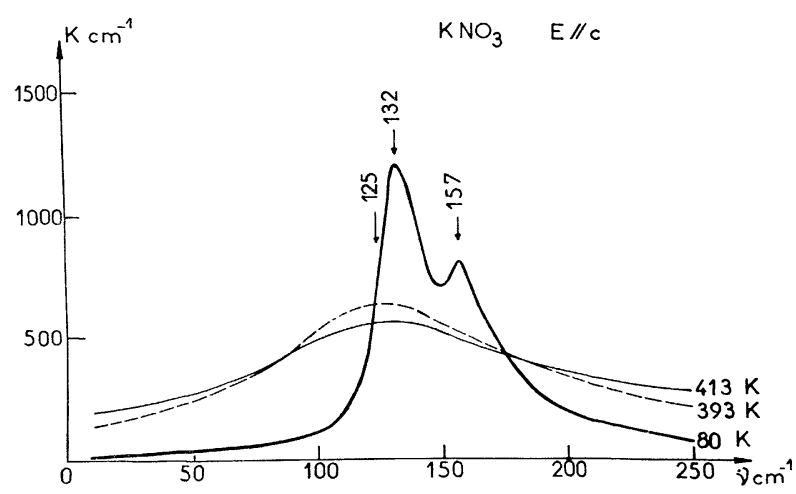

Fig. 1. - Lors de la transition à $398 \mathrm{~K}$ entre les deux phases rhomboédriques I (paraélectrique) et III (ferroélectrique), le spectre de basses fréquences pour $E / / c$ n'est pas modifié et en particulier la bande observée à $125 \mathrm{~cm}^{-1}$ correspondant à la vibration de translation des ions $\mathrm{K}^{+}$et $\mathrm{NO}_{3}^{-}$parallèlement à l'axe $c$ (du type $\mathrm{A}_{1}$ ) ne se comporte pas comme un mode mou. Par ailleurs à $80 \mathrm{~K}$ dans la phase orthorhombique la bande à $125 \mathrm{~cm}^{-1}$ se résout en deux composantes à 132 et $157 \mathrm{~cm}^{-1}$.

\begin{tabular}{|c|c|c|}
\hline $\begin{array}{l}\text { Groupe } \\
\text { d'espace }\end{array}$ & $\begin{array}{c}\text { Nombre } \\
\text { d'unité formulaire } \\
\text { par cellule } \\
\text { élémentaire }\end{array}$ & $\begin{array}{l}\text { Dimensions } \\
\text { de la cellule } \\
\text { éléməntaire en }\end{array}$ \\
\hline- & - & $a=5,94$ \\
\hline $\begin{array}{l}D_{2 b}^{16} \\
P_{n m a} \\
C_{3 v}^{5}\end{array}$ & 4 & $\begin{array}{l}b=9,17 \\
c=6,65\end{array}$ \\
\hline $\mathbf{R}_{3 \mathrm{~m}}$ & 1 & $a=b=c$ \\
\hline $\begin{array}{l}C_{3 v}^{5} \\
R_{3 m}\end{array}$ & 1 & $\begin{aligned} & =4,37 \\
\alpha & =77^{\circ}\end{aligned}$ \\
\hline
\end{tabular}

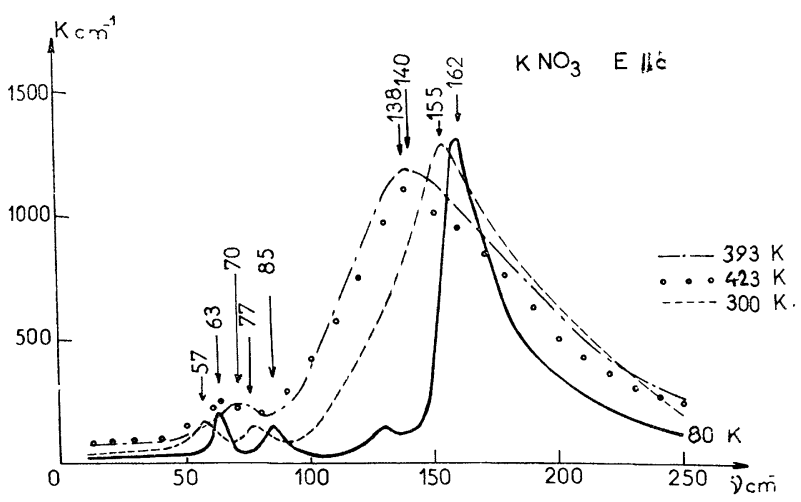

Fig. 2. - Lors de la transition à $398 \mathrm{~K}$ entre les deux phases rhomboédriques I (paraélectrique) et III (ferroélectrique), le spectre de basses fréquences pour $E \perp c$ n'est pas modifié et comporte une bande à $63 \mathrm{~cm}^{-1}$ correspondant à la libration du groupement $\mathrm{NO}_{3}$ autour d'une droite perpendiculaire à l'axe $c$ qui donne une variation de moment dipolaire électrique dans le plan perpendiculaire à $c$ (vibration du type $\mathrm{E}$ ) et une bande beaucoup plus large et beaucoup plus intense à $138 \mathrm{~cm}^{-1}$ correspondant à la vibration de translation des ions $\mathrm{K}^{+}$et $\mathrm{NO}_{3}^{-}$parallèlement au plan perpendiculaire à l'axe $c$ (vibrations du type E). Par ailleurs dès $300 \mathrm{~K}$ dans la phase orthorhombique chacune de ces deux bandes se résout plus ou moins bien en deux composantes. La bande faible à $130 \mathrm{~cm}^{-1}$ provient de la convergence des rayons, se retrouve plus intense pour $E / / c$ et n'est pas significative pour $E \perp c$. Dans cette phase orthorhombique, on observe deux spectres sensiblement identiques pour $E / / a$ et $E / /$

symétrie $C_{3 v}^{5}[5,6,7,10,11]$ (Tableau I), avec une seule unité formulaire par cellule élémentaire. Par suite trois modes de réseau sont possibles en infrarouge et se répartissent dans les deux classes de symétrie $A_{1}$ et $E$ avec deux modes de translation $\left(1 \mathrm{~A}_{1}\right.$ et $\left.1 \mathrm{E}\right)$ et un mode de libration (1 E).

Nous observons effectivement l'activité d'un mode de symétrie $A_{1}$ de fréquence $125 \mathrm{~cm}^{-1}$ (Fig. 1) et de deux modes de symétrie $E$ de fréquence 70 et $140 \mathrm{~cm}^{-1}$ (Fig. 2 et Tableau II) à 393 K. Nous pouvons attribuer les deux vibrations de symétrie $\mathrm{E}$ à $393 \mathrm{~K}$ (Fig. 2 et Tableau II), l'une de fréquence $70 \mathrm{~cm}^{-1}$ au mouvement de libration du groupement $\mathrm{NO}_{3}^{-}$autour d'une droite 
TABLEAU II

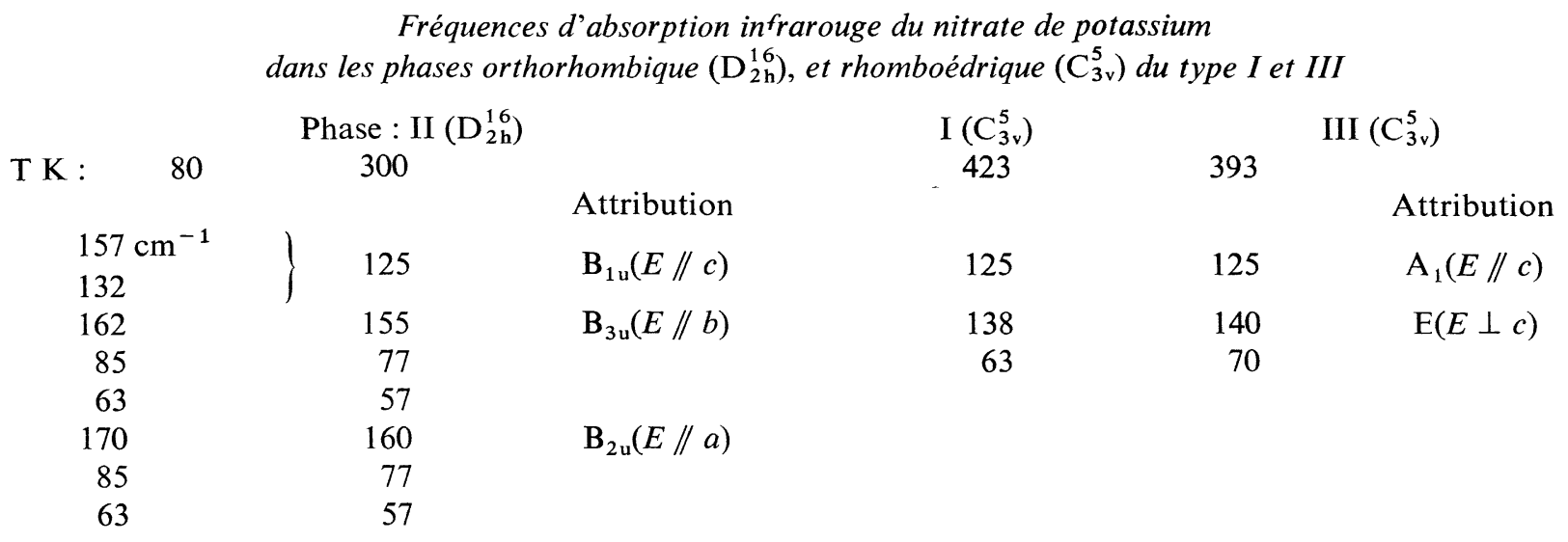

perpendiculaire à l'axe $c$, et l'autre de fréquence $140 \mathrm{~cm}^{-1}$ à la vibration de translation des ions $\mathrm{K}^{+}$et $\mathrm{NO}_{3}^{-}$parallèlement au plan perpendiculaire à l'axe $c$.

Dans la phase II, la cellule élémentaire contient quatre unités formulaires et on attend l'activité de onze modes de réseau en infrarouge, répartis dans les trois classes $B_{1 u}, B_{2 u}$ et $B_{3 u}$ avec sept modes de translation ( $3 \mathrm{~B}_{1 \mathrm{u}}, 1 \mathrm{~B}_{2 \mathrm{u}}$ et $3 \mathrm{~B}_{3 \mathrm{u}}$ ) et quatre modes de libration

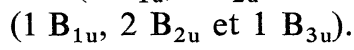

Nous n'observons que huit modes à $80 \mathrm{~K}$ et sept modes à $300 \mathrm{~K}$ sur les onze prévus (Tableau II). D'autre part, contrairement aux prévisions de la théorie des groupes pour la phase II, les modes de fréquence 57 et $77 \mathrm{~cm}^{-1}$ semblent dégénérés puisqu'on les observe dans les deux classes $B_{2 u}$ et $B_{3 u}$ (Tableau II).

L'analyse complète des modes normaux de vibration du nitrate de potassium a été faite par Teng qui a donné une représentation de tous les modes dans chacune des trois phases $[5,6]$.

3.2 Transition entre les PHASes I ET III DE STRUCTURE RHOMBOÉDRIQUE. - Le spectre de vibration de basses fréquences du nitrate de potassium n'est pas modifié lors de la transition à $398 \mathrm{~K}$ entre les deux phases rhomboédriques I (paraélectrique) et III (ferroélectrique) (Fig. 1 et 2 et Tableau II). En particulier la valeur de l'absorption intégrée de 10 à $250 \mathrm{~cm}^{-1}$ : $\int_{10}^{250} \mathrm{~K} \mathrm{~d} \dot{v}$, est voisine dans ces deux phases: $95000 \mathrm{~cm}^{-2}$ et $93000 \mathrm{~cm}^{-2}$ pour $E / / c, 115000 \mathrm{~cm}^{-2}$ et $120000 \mathrm{~cm}^{-2}$ pour $E \perp c$, respectivement dans la phase I et la phase III (l'axe $c$ est l'axe ferroélectrique). Il n'y a donc aucun argument en faveur de l'existence d'un mode mou dans ce domaine de fréquence. Teng met en évidence par diffusion Raman, l'activité d'un mode de symétrie $A_{1}$ à $32 \mathrm{~cm}^{-1}$ dans ces deux phases, ce mode correspondrait au mouvement de vibration de translation des ions $\mathrm{K}^{+}$et $\mathrm{NO}_{3}^{-}$le long de l'axe $c$ [5]. Nous n'avons pas observé l'activité d'un mode de symétrie $\mathrm{A}_{1}$ d'aussi basse fréquence. L'inactivité en absorption (Fig. 1) et en réflexion infrarouge de cette fréquence Raman [10] s'explique d'autant moins s'il s'agit d'un mouvement de translation des ions $\mathrm{K}^{+}$et $\mathrm{NO}_{3}^{-}$le long de l'axe $c$ susceptible de donner une variation importante du moment dipolaire électrique.

3.3 Phase II (Basse température) : Structure ORTHORHOMBIQUE. - Nous avons également étudié le spectre de vibration de la phase II à 300 et $80 \mathrm{~K}$ (Tableau II) ainsi qu'à 10 et 1,3 K.

Dans la structure orthorhombique nous observons deux spectres presque identiques pour l'orientation du champ électrique parallèle à l'axe $a$ et à l'axe $b$ du cristal (Tableau II). La figure 2 ne représente que le spectre correspondant à l'orientation $E / / b$. Seul le mode de plus haute fréquence voit sa dégénérescence levée lorsqu'on passe de la phase III à la phase II, en effet nous observons deux modes distincts de fréquence $155 \mathrm{~cm}^{-1}\left(\mathrm{~B}_{3 \mathrm{u}}\right)$ et $160 \mathrm{~cm}^{-1}\left(\mathrm{~B}_{2 \mathrm{u}}\right)$ à $300 \mathrm{~K}$, qui correspondent au mode de vibration doublement dégénéré de symétrie E observé à $140 \mathrm{~cm}^{-1}$ à $393 \mathrm{~K}$ (phase I, cf. Tableau II), alors que le mode de symétrie $\mathrm{E}$ de fréquence $70 \mathrm{~cm}^{-1}$ à $393 \mathrm{~K}$, donne naissance à deux modes de fréquences 77 et $57 \mathrm{~cm}^{-1}$ à $300 \mathrm{~K}$, actifs dans les deux classes $B_{2 u}$ et $B_{3 u}$. Cette dégénérescence apparente peut être expliquée par le mouvement des ions correspondant à ces modes. Le pivotement des ions nitrate autour d'une direction perpendiculaire à l'axe $c$ produit la même déformation de la cellule élémentaire de la phase II, que le pivotement se produise autour de l'axe $a$ ou de l'axe $b$, alors que le mouvement de translation des ions $\mathrm{K}^{+}$et $\mathrm{NO}_{3}^{-}$met en jeu des forces différentes suivant qu'il se produit parallèlement à l'axe $a$ ou à l'axe $b$, ce qui donne deux modes de fréauence distinctes. L'abaissement de la température à $80 \mathrm{~K}$ ne fait pas apparaître de nouvelles bandes dans les spectres des classes $B_{2 u}$ et $B_{3 u}$ (Tableau II et Fig. 2). Nous avons vérifié qu'il en est de même pour les températures de $10 \mathrm{~K}$ et $1,3 \mathrm{~K}$. Par contre si nous n'observons à $300 \mathrm{~K}$ qu'un seul mode de vibration sur les quatre prévus dans la classe de symétrie $B_{1 u}$, ce mode se dédouble à $80 \mathrm{~K}$ (Fig. 1), il correspond au mouvement de translation des ions $\mathrm{K}^{+}$et $\mathrm{NO}_{3}^{-}$dans une direction parallèle à l'axe $c$. L'abaissement de la température à 10 
et $1,3 \mathrm{~K}$ ne fait apparaître aucun mode de vibration nouveau. Le spectre du nitrate de potassium à ces températures est le même qu'à $80 \mathrm{~K}$.

4. Conclusion. - Le passage de la phase rhomboédrique I à la phase rhomboédrique III (ferroélectrique) ne modifie pas le spectre de vibration qui comporte trois raies externes comme on peut le prévoir pour une cellule élémentaire contenant une seule unité formulaire. La structure rhomboédrique de groupe de symétrie $C_{3 v}^{5}$ dans ces deux phases est donc confirmée par ces résultats.
D'autre part aucun argument pour l'existence d'un mode mou donc pour une transition du type déplacement n'est apporté par notre travail.

Dans la phase orthorhombique nous n'avons observé que sept bandes d'absorption à $300 \mathrm{~K}$ alors que onze vibrations sont théoriquement actives en infrarouge. D'autre part la dégénérescence apparente des modes de symétrie $\mathbf{B}_{2 u}$ et $\mathbf{B}_{3 u}$, interprétée par les mouvements de pivotement des ions nitrate, apporte un argument pour la description de la phase II du nitrate de potassium par une structure orthorhombique pseudo-hexagonale stable jusqu'aux très basses températures.

\section{Bibliographie}

[1] Bridgman, P. W., Proc. Amer. Art. Sci. 51 (1916) 579.

[2] Sawada, S., Nomura, S. et FujII, S., J. Phys. Soc. Japan 13 (1958) 1549.

Sawada, S., Nomura, S. et Asao, Y., J. Phys. Soc. Japan 16 (1961) 2486.

[3] Tahvonen, P. E., Am. Sci. Fermicae Ser. A 1 (1947) 44.

[4] ShinnaKa, Y., J. Phys. Soc. Japan 17 (1962) 1137.

[5] Teng, M. K., Thèse Paris 1969.

Teng, M. K., J. Physique 31 (1970) 771.
[6] Balkanski, M., Teng, M. K. et Nusimovici, M., Phys. Rev. 176 (1968) 1098.

[7] Karpov, S. V. et Shultin, A. A., J. Phys. Chem. Sol. 29 (1968) 475

[8] Yanagi, T., J. Phys. Soc. Japan 20 (1965) 1351.

[9] Nusimovici, M. A., Ferroelectricity (Weller Ed.) 1967, p. 123.

[10] Hill, J. C. et Mohan, P. V., Ferroelectrics 2 (1971) 201.

[11] Brehat, F., Thèse Nancy 1972. 